\title{
DEVELOPMENT OF HIGH EFFICIENT SHELL-AND-TUBE HEAT EXCHANGERS BASED ON PROFILED TUBES
}

\author{
РАЗРАБОТКА ВЫСОКОЭФФЕКТИВНЫХ \\ КОЖУХОТРУБНЫХ ТЕПЛООБМЕННИКОВ \\ НА ОСНОВЕ ПРОФИЛИРОВАННЫХ ТРУБ
}

\section{Djamalutdin Chalaev ${ }^{1}$ \\ Nina Silnyagina ${ }^{2}$}

DOI: https://doi.org/10.30525/978-9934-26-021-6-42

\begin{abstract}
The use of advanced heat transfer surfaces (corrugated tubes of various modifications) is an effective way to intensify the heat transfer and improve the hydraulic characteristics of tubular heat exchangers. The methods for evaluating the use of such surfaces as working elements in tubular heat exchangers have not been developed so far. The thermal and hydrodynamic processes occurring in the tubes with the developed surfaces were studied to evaluate the efficiency of heat exchange therein. Thinwalled corrugated flexible stainless steel tubes of various modifications were used in experimental studies. The researches were carried out on a laboratory stand, which was designed as a heat exchanger type «tube in tube» with a corrugated inner tube. The stand was equipped with sensors to measure the thermal hydraulic flow conditions. The comparative analysis of operation modes of the heat exchanger with a corrugated inner tube of various modifications and the heat exchanger with a smooth inner tube was performed according to the obtained data. Materials and methods. A convective component of the heat transfer coefficient of corrugated tube increased significantly at identical flow conditions comparing with a smooth tube. Increasing the heat transfer coefficient was in the range of 2.0 to 2.6 , and increased with increasing Reynolds number. The increase in heat transfer of specified range outstripped the gain of hydraulic resistance
\end{abstract}

\footnotetext{
${ }^{1}$ Ph.D., Leading Researcher of Department Heat and Mass Transfer in Disperse Systems, Institute of Engineering Termophysics of National Academy of Sciences of Ukraine, Ukraine ${ }^{2}$ Senior Researcher of Department Heat and Mass Transfer in Disperse Systems, Institute of Engineering Termophysics of National Academy of Sciences of Ukraine, Ukraine 
caused by increase of the flow. Results and discussion. CFD model in the software ANSYS CFX 14.5 was adapted to estimate the effect of the tube geometry on the intensity of the heat transfer process. A two-dimensional axially symmetric computer model was used for the calculation. The model is based on Reynolds equation (Navier-Stokes equations for turbulent flow), the continuity equation and the energy equation supplemented by the conditions of uniqueness. SST-turbulence model was used for the solution of the equations. The problem was solved in the conjugate formulation, which allowed assessing the efficiency of heat exchange, depending on various parameters (coolant temperature, coolant velocity, pressure). The criteria dependences were obtained $\mathrm{Nu}=\mathrm{f}(\mathrm{Re}, \mathrm{Pr})$. Conclusions. The use a corrugated tube as a working element in tubular heat exchangers can improve the heat transfer coefficient of 2.0-2.6 times, with an increase in hydraulic resistance in the heat exchanger of 2 times (compared with the use of smooth tubes). The criteria dependences obtained on the basis of experimental studies and mathematical modeling allow developing a methodology for engineering calculations for the design of new efficient heat exchangers with corrugated tubes.

\section{1. Вступление}

Во всем мире и Украине развитие энергетики происходит в условиях роста стоимости энергоносителей. Чрезмерное потребление энергоресурсов в значительной степени ограничивает возможности экономического потенциала каждой страны. Для реализации планов энергосбережения необходимо разработка нового высокоэкономичного энергооборудования и модернизация морально и физически устаревших теплообменных аппаратов.

Теплообменные аппараты занимают важное место в промышленной теплоэнергетике и составляют большую группу теплосилового оборудования, занимая значительные производственные площади и превышая чаще всего $20 \%$ стоимости общей комплектации в теплоэнергетике, химической, нефтеперерабатывающей, пищевой промышленности, ЖКХ и ряде других отраслей.

В энергетических устройствах и технологической аппаратуре большую роль играют тепломассообменные процессы. Повышение эффективности этих процессов и совершенствование теплообменного 
оборудования является важной составляющей вопроса рационального использования энергии. Серийно выпускаемые пластинчатые и кожухотрубные теплообменники имеют ряд недостатков. Кожухотрубные теплообменники относительно недорогие, работают в широком диапазоне температур и давлений, однако, они имеют низкие коэффициенты теплопередачи и высокую металлоемкость. В последние годы наблюдается тенденция вытеснения трубчатых теплообменных аппаратов пластинчатыми, у которых коэффициенты теплопередачи значительно выше, но они имеют также ряд недостатков, в числе которых высокая стоимость изготовления, склонность к засорению взвешенными частицами, высокая зависимость теплогидравлических показателей от накипных отложений. В связи с этим актуальной является задача повышения эффективности работы трубчатых теплообменных аппаратов (рис. 1).

Дальнейшее совершенствование теплообменного оборудования требует поиска новых методов интенсификации теплопередачи. Одним из способов повышения эффективности этих аппаратов является интенсификация теплоотдачи с помощью различного рода турбулизаторов потока. К ним относятся трубы с ленточными вставками, внутренним оребрением, локальными завихрителями потока, полусферическими лунками и др. Для увеличения интенсивности теплообмена в межтрубном пространстве применяют спиральные ребра, проволочное оребрение, а также переход с продольного обтекания пучков труб на поперечный с помощью винтообразных перегородок [1; 2].

При выборе для практического применения того или иного метода интенсификации теплообмена приходится учитывать не только эффективность самой поверхности, но и ее универсальность для одно- и двухфазных теплоносителей, технологичность ее изготовления, технологичность сборки теплообменного аппарата, прочностные требования, загрязняемость поверхности, особенности эксплуатации и т.д. Все эти особенности существенно снижают возможности выбора эффективных поверхностей.

Анализ различных методов интенсификация теплообмена показывает, что большинство из них вызывают турбулизацию потока по всему сечению трубы - как в пристенном слое, так и центральной зоне. Вследствие этого рост затрат энергии на преодоление гидравли- 
Djamalutdin Chalaev, Nina Silnyagina

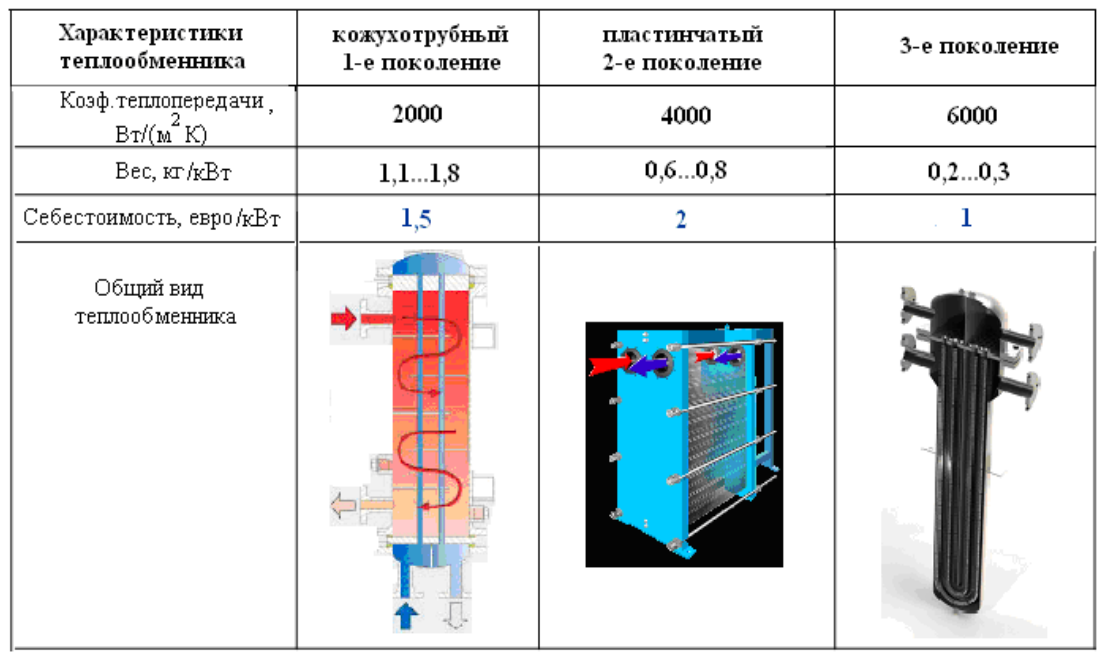

Рис. 1. Общие характеристики существующих теплообменников

ческого сопротивления превышает рост теплоотдачи. Так, при увеличении теплопередачи через стенку трубы в 2-3 раза гидравлическое сопротивление системы растет в 10-12 раз.

С этой точки зрения наиболее эффективным является метод турбулизации пристенного потока. Установлено, что накатка на трубе кольцевых канавок с определенным шагом и глубиной позволяет турбулизовать только пристенный ламинарный слой, поэтому затраты энергии на прокачку теплоносителя увеличиваются незначительно - при росте коэффициента теплопередачи в 2 раза гидравлическое сопротивление увеличивается только в 2,5 раза. Обнаруженная закономерность опережающего роста теплоотдачи в каналах с дискретными турбулизаторами относительно роста гидравлического сопротивления по сравнению с аналогичным гладким каналом была признана научным открытием [3].

Создание современного теплообменного оборудования - задача сложная и многоплановая. Необходимость сокращения расхода энергии и материалов, а также снижение стоимости теплообменного оборудования обусловила расширение работ, направленных на 
интенсификацию процесса теплообмена, снижение массы и габаритов теплообменников, увеличения их тепловой производительности или снижения затрат энергии на осуществление процессов теплопередачи. Однако, на сегодняшний день считаются почти исчерпанными резервы реальной рационализации конструкций теплообменников.

Для улучшения характеристик теплообменного оборудования необходимо разрабатывать новые конструкции теплообменных аппаратов, увеличивая эффективность теплообменных поверхностей. Основной задачей при создании эффективного теплообменного аппарата является необходимость выдержать заданные значения по количеству передаваемой теплоты, гидравлическому сопротивлению, и при этом, сделать его как можно более компактным и легким. Выполнить эти противоречивые требования, возможно только используя интенсификацию теплообмена.

Интенсификация процессов теплообмен - одно из важнейших направлений развития современного теплообменного оборудования. Интенсификация процессов теплообмена обычно не представляет интереса, если не дает выигрыша в стоимости по сравнению с использованием стандартных образцов. На выбор метода интенсификации дополнительно влияют такие факторы, как наличие материалов, безопасность и надежность. Примерная взаимосвязь свойств теплообменников при их оценке и сравнению представлены на рис. 2 [4-7].

В поиске методов интенсификации теплообменных процессов постоянно проводятся исследования зарубежных и отечественных ученых. В настоящее время наиболее широко применяют пассивные методы интенсификации теплообмена и, в частности, использование развитых поверхностей теплообмена. До настоящего времени не разработаны универсальные методы и критерии для оценки эффективности поверхностей теплообмена.

Необходимо дальнейшее углубленное изучение тепловых и гидродинамических процессов, протекающих в развитых поверхностях теплообмена, с целью получения обобщенных зависимостей для расчета тепловых и гидравлических характеристик и оценки их эффективности. 
Djamalutdin Chalaev, Nina Silnyagina

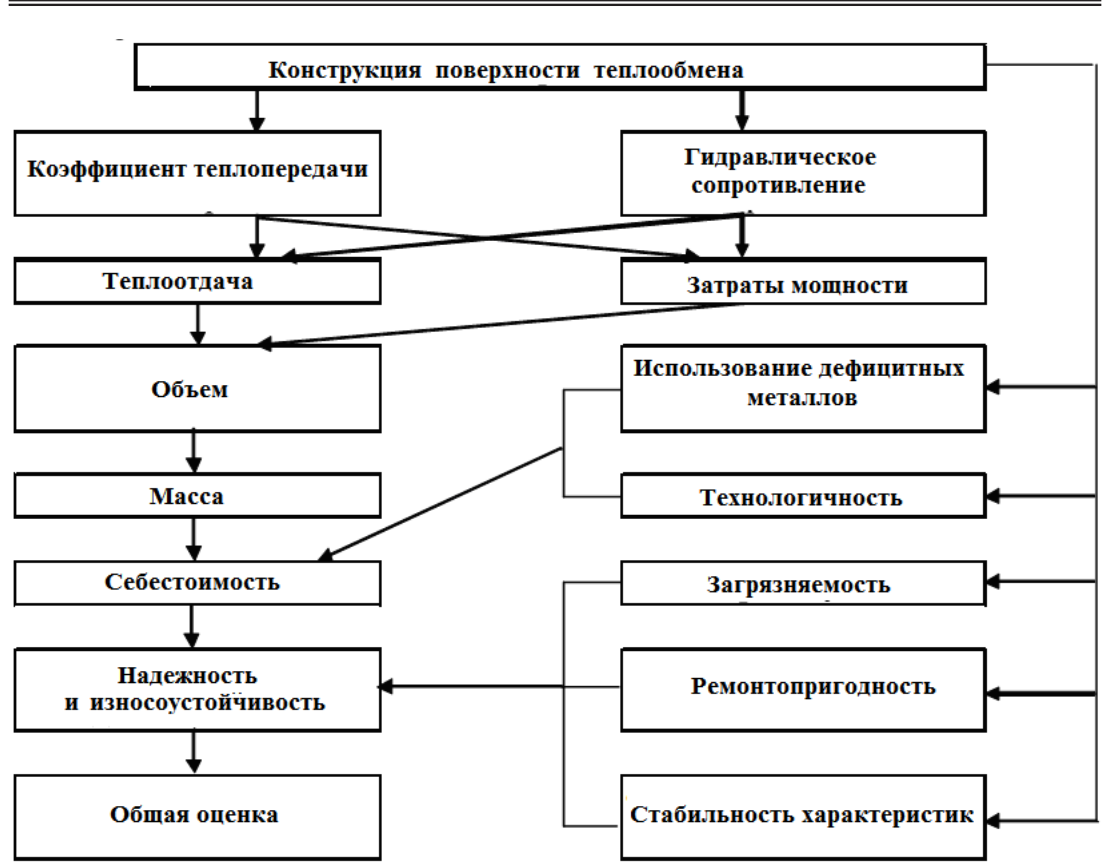

Рис. 2. Взаимосвязь свойств теплообменников при их оценке и сравнении

\section{2. Материалы и методы}

Перспективным направлением повышения эффективности теплообменной поверхности является применение тонкостенных гибких нержавеющих профилированных труб, способствующих формированию вихревой структуры потока, направленной к стенке трубы, что обеспечивает опережающий рост теплообмена по сравнению с увеличением сопутствующих потерь давления.

В последние годы отечественной промышленностью освоено производство гибких нержавеющих профилированных труб небольшого диаметра (рис. 3). Эти трубы широко применяются для монтажа трубопроводов горячей и холодной воды, устройств теплых полов и стен, подведению газа к газопотребляющему оборудованию. Такая теплообменная поверхность характеризуется компактностью, невысокой удельной 


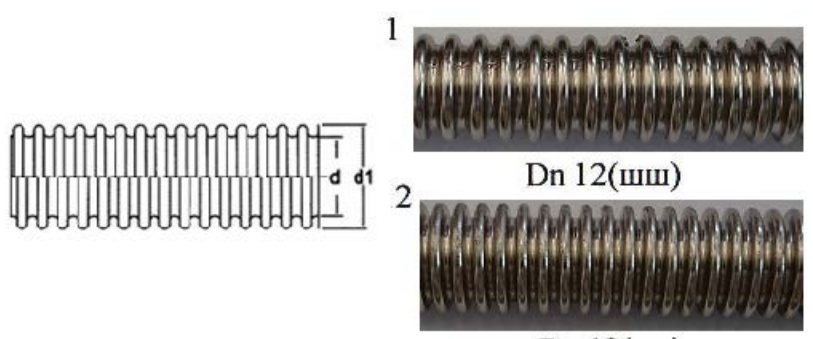

Dn 12(cw)

Рис. 3. Тонкостенные гофрированные трубы различных модификаций: 1 - профилированная труба диаметром 12 с широким шагом гофр; 2 - профилированная труба диаметром 12 со средним шагом гофр

стоимостью, возможностью создания сложных теплообменных поверхностей за счет изгиба и закрутки пучка профилированных труб.

По данным фирм-производителей гофрированные трубы не боятся разморозки и гидравлических ударов, самостоятельно компенсируют линейные расширения и могут работать при температурах до $150{ }^{\circ} \mathrm{C}$ и давлении до 25 кг/см². Такие трубы характеризуются долговечностью за счет коррозионной стойкости и высокой прочности труб, надежностью и устойчивостью к внешним и внутренним механическим и гидравлическим нагрузкам за счет свойств материала, высокой гибкостью и упругостью $[3 ; 10]$.

Использование гибких гофрированных труб различных модификаций позволяет улучшить теплогидравлические характеристики теплообменников. Гофрированные трубы обеспечивают турбулентность вблизи поверхности стенки трубы, тем самым уменьшает толщину теплового пограничного слоя. В результате улучшается перемешивание жидкости вблизи стенки трубы, благодаря отрывным возмущением от стенки к основному потоку, тем самым происходит увеличение общего коэффициента теплопередачи в теплообменных системах (рис. 4).

Однако, в настоящее время в литературе практически отсутствуют надежные данные по теплообмену и гидравлическому сопротивлению гибких гофрированных труб, что затрудняет проектирование теплообменных аппаратов и оптимизацию их параметров. Для решения дан- 


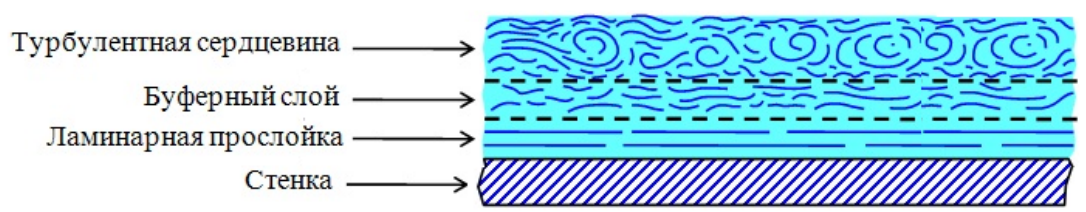

a)

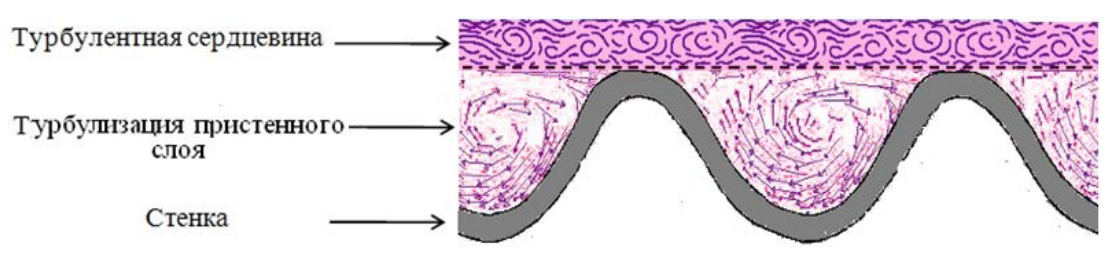

б)

\section{Рис. 4. Схема формирования потоков в трубах с различной геометрией: а) гладкая труба; б) профилированные трубы}

ной задачи в ИТТФ НАН Украины был создан стенд (рис. 5) и проведены экспериментальные исследования интенсивности теплопередачи при использовании тонкостенных гофрированных труб различных модификаций [8].

\section{3. Результаты и обсуждение}

На рис. 6-7 приведены результаты исследований и получены зависимости коэффициента теплопередачи от скорости теплоносителя в гофрированных трубах по сравнению с гладкой трубой. Проводился сравнительный анализ режимов работы теплообменника с гофрированной внутренней трубой различных модификаций и теплообменника с гладкой внутренней трубой. По сравнению с гладкой трубой, конвективная составляющая коэффициента теплопередачи гофрированной трубы значительно увеличивается, при одинаковых условиях потока. Повышение коэффициента теплопередачи оказалось в диапазоне от 2,0 до 2,6, и увеличивалось с увеличением числа Рейнольдса. Прирост теплопередачи, в указанном диапазоне, опережает прирост гидравлического сопротивления обусловленного увеличением скорости потока. 


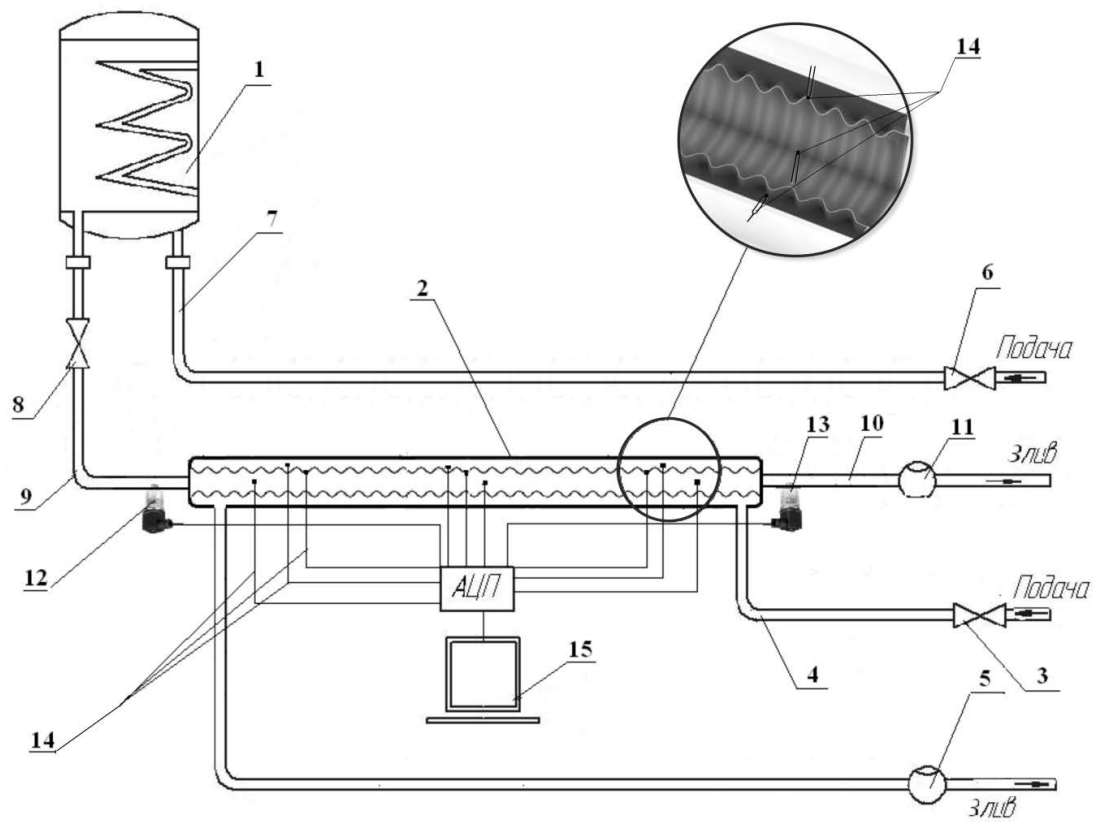

Рис. 5. Принципиальная схема лабораторного стенда для экспериментальных исследований процесса теплопередачи в теплообменниках на основе профилированных труб:

1 - теплоизолированный бойлер; 2 - теплообменник типа «труба в трубе»;

3 - вентиль подачи холодного теплоносителя; 4 - трубопровод подачи холодного теплоносителя; 5 - счетчик расхода холодного теплоносителя;

6 - вентиль подачи холодной воды в бойлер; 7 - трубопровод подачи холодной воды в бойлер; 8 - вентиль подачи горячего теплоносителя; 9 - трубопровод подачи горячего теплоносителя; 10 - трубопровод слива горячего теплоносителя; 11 - счетчик расхода горячего теплоносителя; 12,13 - датчики (фирмы DANFFOS) для измерения давления на входе и выходе трубного пространства теплообменника; 14 - термопары хромель-копель (ХК); 15 - персональный компьютер

Исследования показали, что гофрированные трубы эффективно турбулизируют поток в пристенной зоне течения, разрушая эту область с помощью отрывных возмущений потока, которые генерируются тур- 
булизаторами и обеспечивают высокие коэффициенты теплопередачи сравнительно с гладкими трубами.

Было изучено влияние геометрии труб на интенсивность процесса теплообмена, что позволило провести оценку эффективности теплопередачи в зависимости от различных параметров.

Кроме этого, для оценки влияния геометрии труб на интенсивность процесса теплообмена была адаптирована CFD модель в программной среде ANSYS CFX 14.5. Для расчетов использовалась двухмерная осесимметричная компьютерная модель. В основу модели положены уравнения Рейнольдса (уравнение Новье-Стокса для турбулентного потока), уравнение неразрывности и уравнения энергии дополнены условиями однозначности. Для замыкания уравнений использовалась SST-модель турбулентности. Задача решалась в сопряженной постановке, что позволяет проводить оценку эффективности теплообмена

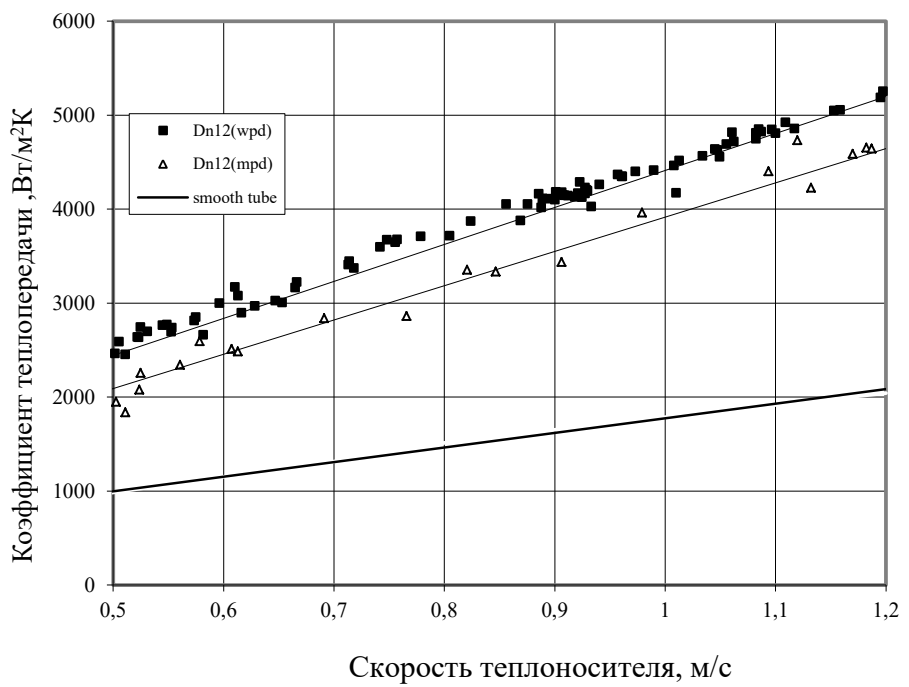

Рис. 6. Зависимость коэффициента теплопередачи для гофрированных и гладких трубы от скорости движения теплоносителя: 1 - профилированная труба диаметром 12 с широким шагом гофр; 2 - профилированная труба диаметром 12 со средним шагом гофр; 3 - гладкостенная труба 


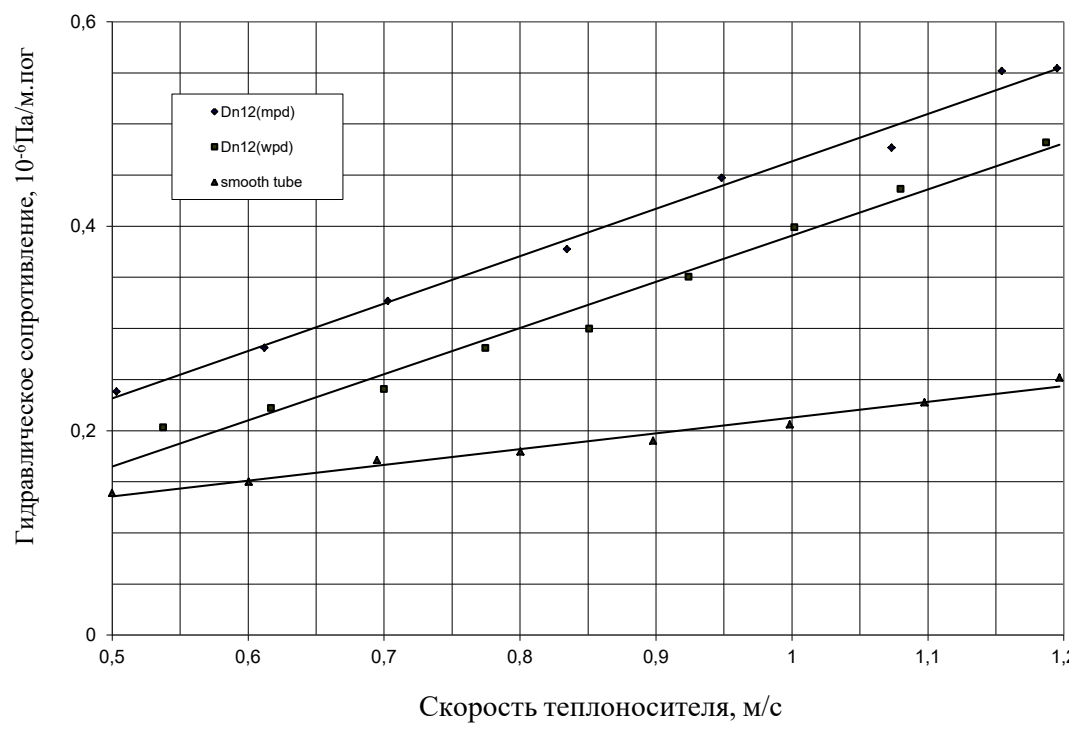

Рис. 7. Зависимость гидравлического сопротивления для гофрированных и гладких трубы от скорости движения теплоносителя: 1 - профилированная труба диаметром 12 с широким шагом гофр; 2 - профилированная труба диаметром 12 со средним шагом гофр; 3 - гладкостенная труба

в зависимости от различных параметров (температура теплоносителя, скорость теплоносителя, давление).

При обобщении экспериментальных данных для внутреннего канала профилированной трубы в качестве базового использовалось уравнение подобия степенного типа для течения в трубах:

$$
\overline{N u_{d}}=C \cdot \operatorname{Re}_{d}^{m} \cdot \operatorname{Pr}_{\varkappa}^{0,43} \cdot\left(\frac{\operatorname{Pr}_{ж}}{\operatorname{Pr}_{c m}}\right)^{0,25},
$$

из которого статистической обработкой экспериментальных данных определялись значения константы $C$ и показателя степени $m$.

Для этого экспериментальные данные представлялись в логарифмической системе координат: 


$$
F^{*}=\lg \left(\frac{\overline{N u_{ж}}}{\operatorname{Pr}_{f}^{0.43} \cdot\left(\operatorname{Pr}_{f} / \operatorname{Pr}_{w}\right)^{0.25}}\right)=\lg C+m \lg \left(\operatorname{Re}_{d}\right)
$$

в которой опытные данные располагаются около прямой (рис. 8) с константой $C$ в уравнении (2) равной 0,79 и показателем степени $m=0,66$.

Значение показателя степени 0,66 обычно соответствует переходному режиму. Поскольку опыты проведены при числах Рейнольдса более 9000, то можно считать, что поток в профилированной трубе был турбулентным. Поэтому была выполнена дополнительная обработка опытных данных с показателем степени при числе Рейнольдса в уравнении (1) равным 0,8 .

Как следует из рис. 9, в представленной системе координат константа $C$ не является постоянной, а зависит от числа Рейнольдса и определяется уравнениями:

$$
\lg (C)=-0.02 \cdot \lg \left(\operatorname{Re}_{d}\right)+0,12
$$

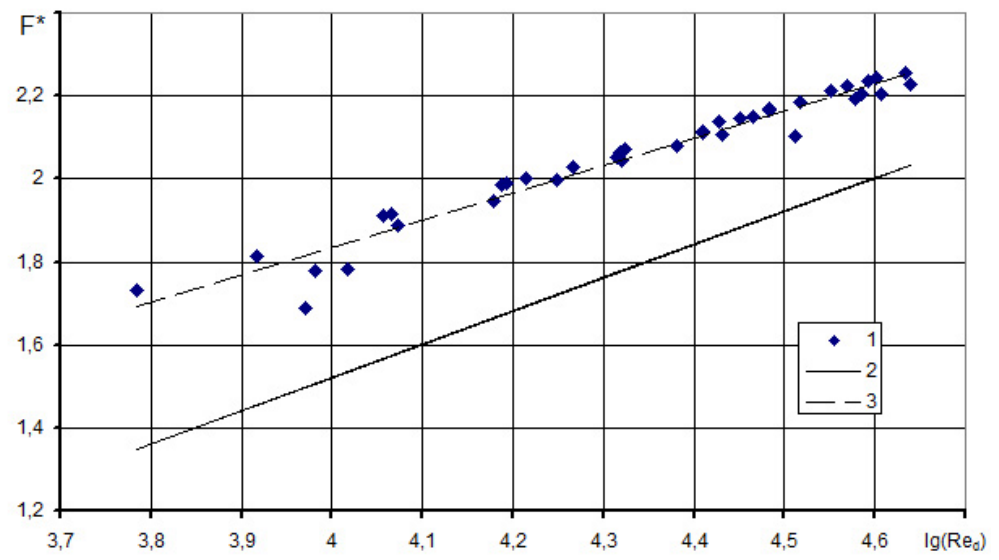

Рис. 8. Результаты обобщения опытных данных по среднему теплообмену внутри профилированной трубы: 1 - опытные данные; 2 - уравнение для среднего теплообмена в трубе на участке стабилизированного течения $\overline{N u_{d}}=0,021 \cdot \operatorname{Re}_{d}^{0,8} \cdot \operatorname{Pr}_{{ }^{0}}^{0.43}$; 3 - аппроксимация экспериментальных данных 


$$
C=10^{-0.02 \cdot \lg \left(\operatorname{Re}_{d}\right)+0.12}
$$

В этом случае уравнение для среднего теплообмена внутри профилированного канала принимает следующий вид:

$$
\overline{N u_{d}}=10^{-0.02 \cdot \lg \left(\operatorname{Re}_{d}\right)+0.12} \cdot \operatorname{Re}_{d}^{0.8} \cdot \operatorname{Pr}_{;}^{0.43} \cdot\left(\frac{\operatorname{Pr}_{\varkappa}}{\operatorname{Pr}_{c m}}\right)^{0,25}
$$

На рис. 9 использовано:

$$
F^{* *}=\lg \left(\frac{\overline{N u_{d}}}{\operatorname{Pr}_{f}^{0,43} \cdot\left(\operatorname{Pr}_{f} / \operatorname{Pr}_{w}\right)^{0,25} \cdot \operatorname{Re}_{d}^{0,8}}\right)
$$

Аналогичное обобщение опытных данных было выполнено для среднего теплообмена в кольцевом канале. Результаты обобщения опытных данных в полулогарифмической системе координат с определением константы $C$ и показателя степени $m$ представлены на рис. 10 . В этом случае константа $C$ равна 1,77 , а показатель степени $m=1,0$.

Однако значение показателя степени $\mathrm{m}$ не соответствуют физической картине теплообмена в кольцевом канале поскольку опыт-

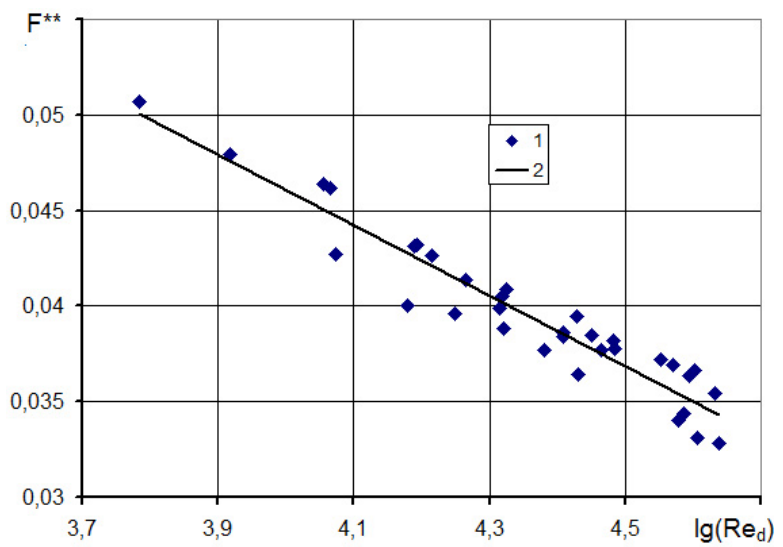

Рис. 9. Результаты обобщения опытных данных

по среднему теплообмену для внутреннего канала профилированной трубы с показателем степени 0,8 при числе

Рейнольдса: 1 - опытные данные; 2 - обобщающая линия 


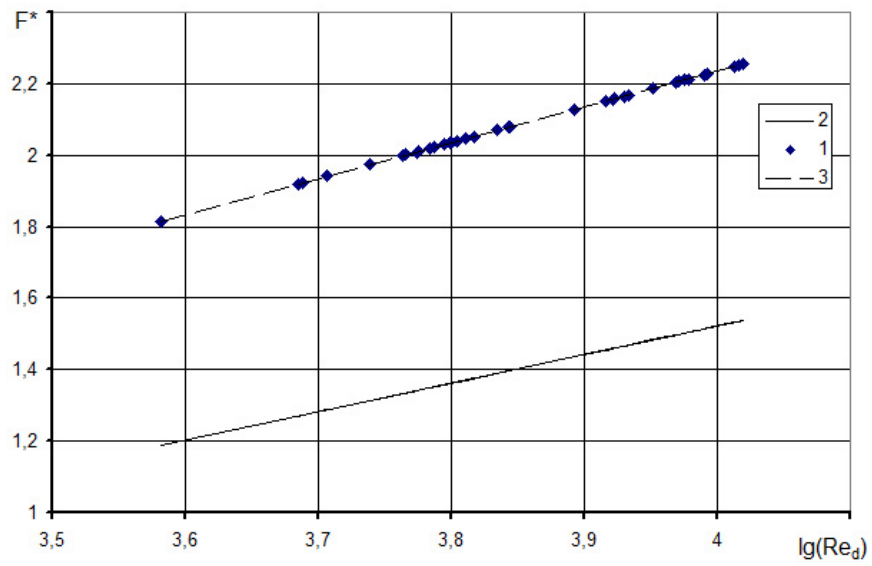

Рис. 10. Результаты обобщения опытных данных для среднего теплообмена в кольцевом канале: 1 - опытные данные;

2 - уравнение для среднего теплообмена в трубе на участке стабилизированного течения $\overline{N u_{d}}=0,021 \cdot \operatorname{Re}_{d}^{0,8} \cdot \operatorname{Pr}^{0.43}$;

\section{3 - обобщающая линия}

ные данные получены при числе Рейнольдса более 4000. Несмотря на малое значение числа Рейнольдса, соответствующее переходному режиму при осевом течении, можно предполагать, что в данном случае в кольцевом канале существует турбулентный режим. Поэтому было выполнено обобщение опытных данных с показателем степени при числе Рейнольдса равным 0,8 . Результаты такого обобщения опытных данных представлены на рис. 11.

В представленной системе координат уравнение для константы C будет определяться зависимостями:

$$
\begin{aligned}
\lg (C) & =0.05 \cdot \lg \left(\operatorname{Re}_{d}\right)-0,08 \\
C & =10^{0.05 \cdot \lg \left(\mathrm{Re}_{d}\right)-0.08}
\end{aligned}
$$

а уравнение для среднего теплообмена в кольцевом канале принимает следующий вид:

$$
\overline{N u_{d}}=10^{0.05 \cdot \lg \left(\mathrm{Re}_{d}\right)-0,08} \cdot \operatorname{Re}_{d}^{0.8} \cdot \operatorname{Pr}_{;}^{0.43} \cdot\left(\frac{\operatorname{Pr}_{\mathcal{F}}}{\operatorname{Pr}_{c m}}\right)^{0,25}
$$




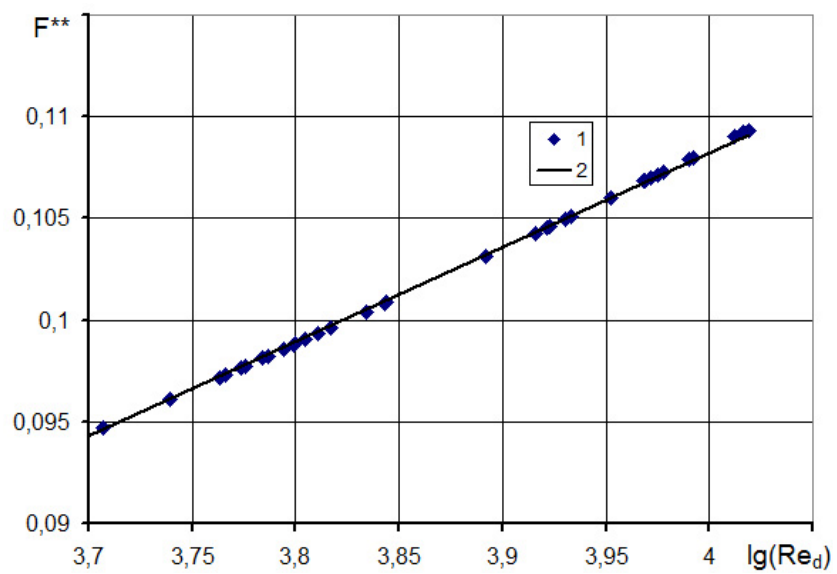

Рис. 11. Результаты обобщения опытных данных для среднего теплообмена в кольцевом канале профилированной трубы с показателем степени 0,8 при числе Рейнольдса: 1 - опытные данные; 2 - обобщающая линия

Результаты показали, что математические расчеты достаточно близки с экспериментальными исследованиями. Полученные критериальные зависимости позволяют разработать методику инженерных расчетов для проектирования новых эффективных теплообменников в которых в качестве теплообменных элементов используются тонкостенные гибкие нержавеющие профилированные трубы.

Теоретические и экспериментальные исследования позволили создать эффективный кожухотрубный теплообменник, в котором в качестве теплообменных элементов используются тонкостенные гибкие нержавеющие профилированные трубы (рис. 12). Теплотехнические испытания экспериментального образца нового теплообменника были проведены на тепловом пункте административного здания ИТТФ НАН Украины (рис. 13). Работа теплообменника на протяжении отопительного сезона в составе теплового пункта показала, что средний коэффициент теплопередачи в новом теплообменнике составил около 6000 Вт/(м²·К). Общие характеристики нового эффективного кожухотрубного теплообменника на основе профилированных труб представлены в таблице 1. 
При изготовлении теплообменника была решена задача крепления труб к трубной решетке, так как традиционные методы вальцевания и сварки из-за специфически малой толщины стенки труб ( $\delta=0,3$ мм) не подходят [9]. Для сборки теплообменного аппарата был разработан и изготовлен специальный инструмент, который позволяет за один прием зафиксировать и запрессовать трубу в трубной решетке (рис. 14).

Сравнительные теплотехнические испытания нового теплообменника показали:

- использование в составе индивидуального теплового пункта нового теплообменника на основе профилированных труб обеспечи-

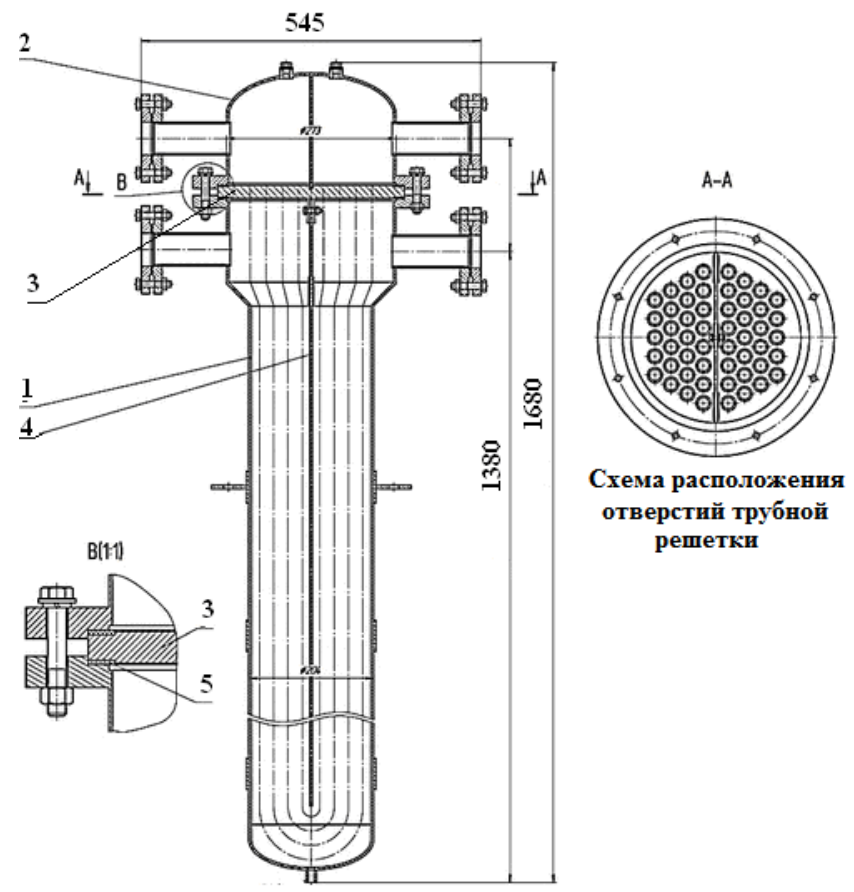

Рис. 12. Общий вид нового эффективного теплообменника на основе профилированных труб: 1 - корпус теплообменника; 2 -крышка теплообменника; 3 - трубная решетка; 4 - перегородка; 5 - прокладки 


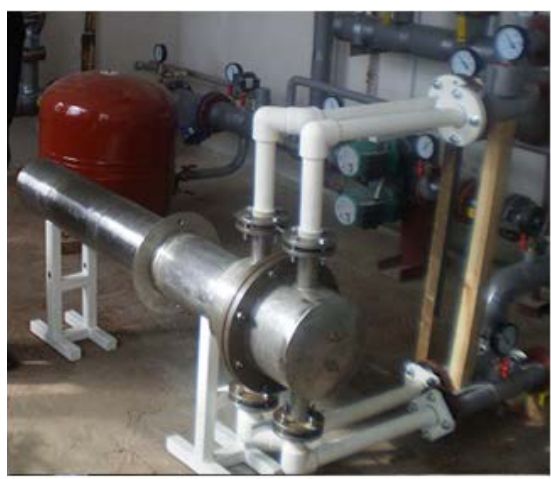

a)

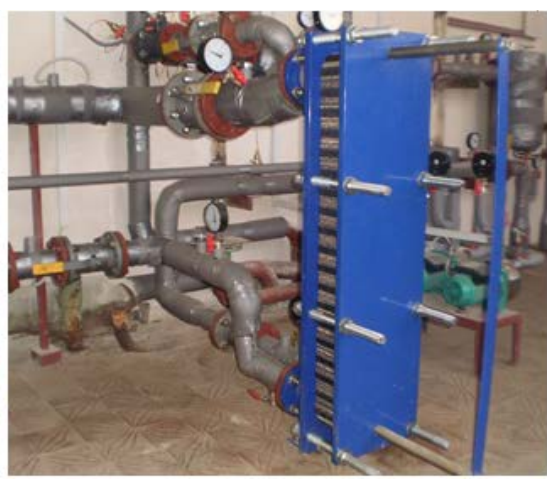

6)

Рис. 13. Сравнительные испытания нового эффективного теплообменника на основе профилированных труб (а) и пластинчатого теплообменника фирмы Альфа Лаваль (б) на ТП

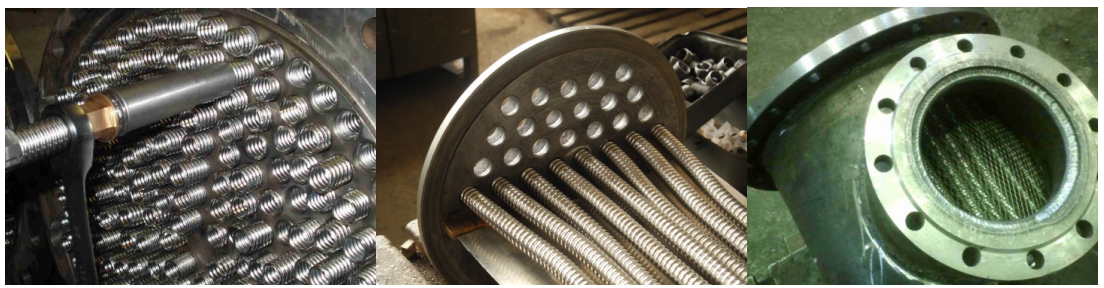

Рис. 14. Кожухотрубный теплообменный аппарат с профилированными трубами на этапе крепления труб к трубной решетке

вает эффективную работу теплового пункта во всем диапазоне изменения отопительной нагрузки;

- оснащение индивидуальных тепловых пунктов новым теплообменником на основе профилированных труб позволит на 20-30\% уменьшить затраты на их изготовление;

- для оснащения типовых тепловых пунктов теплообменниками на основе профилированных труб необходимо разработать типовой ряд конструкций таких теплообменных аппаратов различной мощности. 
Таблиця 1

Общие характеристики нового эффективного кожухотрубного теплообменнка на основе профилированных труб

\begin{tabular}{|c|c|c|c|c|}
\hline \multirow{2}{*}{$\begin{array}{c}\text { Площадь } \\
\text { поверхности } \\
\text { ТО, } \text { м }^{2}\end{array}$} & \multicolumn{2}{|c|}{ Параметры среды } & \multirow{2}{*}{$\begin{array}{c}\text { Общая } \\
\text { маса ТО, } \\
\text { кг }\end{array}$} & \multirow{2}{*}{$\begin{array}{c}\text { Коэффициенты } \\
\text { теплопередачи } \\
\text { в эталонном } \\
\text { режиме Вт/м² К }\end{array}$} \\
\hline & $\mathrm{t},{ }^{\circ} \mathrm{C}$ & 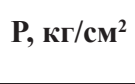 & & \\
\hline 4,5 & 95 & 10 & 80 & 6000 \\
\hline
\end{tabular}

\section{4. Выводы}

Получены критериальные зависимости для расчета и оптимизации теплопереноса в трубчатом теплообменнике на основе промышленно выпускаемых гибких гофрированных нержавеющих труб. Применение таких труб позволило достичь высоких значений коэффициента теплопередачи в трубчатом теплообменнике. Испытания теплообменника показали, что эффективная турбулизация потока теплоносителя и создание режима автоколебаний теплообменных трубок при движении теплоносителя способствуют предупреждению образования отложений на стенках трубок, благодаря чему решается актуальная проблема загрязнения и очистки. Возможна эксплуатация теплообменников такого типа без операций очистки в течение всего периода работы, так как толщина загрязнений в профилированных трубках после определенного времени работы практически стабилизируется на допустимом уровне. Благодаря пружинящим свойствам гибкая профилированная труба нечувствительна к температурным расширениям и гидравлическим ударам. Теплообменник в процессе испытаний показал эффективную работу во всем диапазоне изменения тепловых нагрузок. Разработанный теплообменник имеют почти в 1,5 раза меньшую металлоемкость по сравнению с пластинчатым теплообменником аналогичной мощности. Стоимость единицы теплообменной поверхности кожухотрубных теплообменников на основе профилированных труб ниже, чем аналогичные показатели пластинчатых теплообменников, что является существенным резервом для удешевления такого типа теплообменного оборудования. Оснащение индивидуальных тепловых пунктов новым теплообменником позволит на 20-30\% уменьшить затраты на их изготовление. Применение нового метода крепления труб к трубной решетке дает возможность снизить стоимость изготовления теплообменника в целом и возможность без проблем заменять трубы в процессе эксплуатации аппарата. 


\section{Список литературы:}

1. Kuppan Thulukkanam (2013). Heat Exchanger Design Handbook, Second Edition. CRC Press, Suite 300Boca Raton.

2. Дрейцер Г.А. Проблемы создания компактных трубчатых теплообменных аппаратов. Теплоэнергетика. 1995. № 3. С. 11-18.

3. Калинин Э.К., Дрейцер Г.А., Ярхо С.А. и др. Закономерность изменения теплоотдачи на стенках каналов с дискретной турбулизацией потока при вынужденной конвекции (диплом на открытие № 242, СССР). Открытия, изобретения. 1981. № 35. С. 3.

4. M. Pan, S. Jamaliniya, R. Smith, I. Bulatov, M. Gough, T. Higley, P. Droegemueller. New insights to implement heat transfer intensification for shell and tube heat exchangers. Energy. Volume 57. 1 August 2013. P. 208-221.

5. Hasanpour A., Farhadi M., Sedighi K. (2016). Experimental heat transfer and pressure drop study on typical, perforated, V-cut and U-cut twisted tapes in a helically corrugated heat exchanger. International Communications in Heat and Mass Transfer. No. 71. Pp. 126-136.

6. Seong-Yeon Yoo, Dong-Seong Park, Min-Ho Chung, Sang-Yun Lee (2002). Heat transfer enhancement for fin-tube heat exchanger using vortex generators, Journal of Mechanical Science and Technology. No. 16. Pp. 109-115.

7. Seong Won Hwang, Dong Hwan Kim, June Kee Min, Ji Hwan Jeong (2012). CFD analysis of fin tube heat exchanger with a pair of delta winglet vortex generators, Journal of Mechanical Science and Technology. No. 26. Pp. 2949-2958.

8. D. Chalaev, N. Silnyagina, O. Shmatok, A. Nedbailo. Heat transfer enhancement in a corrugated tube heat exchanger. Ukrainian Food Journal. 2016. Vol. 5. Issue 2. P. 376-386.

9. D. Chalaev, O. Shmatok, T. Grabova, N. Silnyagina. Development of energyefficient shell and tube heat exchangers for use in geothermal heating systems. $2^{\text {nd }}$ International scientific conference "Chemical technology and engineering". Lviv, June 24-28 ${ }^{\text {th }}$, 2019. P. 170-172.

10. Available at: http://www.sanzee.com

\section{References:}

1. Kuppan Thulukkanam (2013). Heat Exchanger Design Handbook, Second Edition, CRC Press, Suite 300Boca Raton.

2. Dreytser G.A. (1995). Problemy sozdaniya kompaktnyih trubchatyih teploobmennyih apparatov [Problems of creating compact tubular heat exchangers]. Teploenergetika, no. 3, pp. 11-18.

3. Kalynyn E.K., Drejcer G.A., Jarkho S.A. y dr. (1981). Zakonomernostj izmenenyja teplootdachy na stenkakh kanalov s dyskretnoj turbulyzacyej potoka pry vinuzhdennoj konvekcyy (diplom na otkritye № 242, SSSR) [Regularity of changes in heat transfer on the walls of channels with discrete flow turbulization during forced convection (diploma for opening No. 242, USSR)]. Otkrityja, yzobretenyja, no. 35 , p. 3 . 
4. Pan M., Jamaliniya S., Smith R., Bulatov I., Gough M., Higley T., Droegemueller P. (2013). New insights to implement heat transfer intensification for shell and tube heat exchangers. Energy, no. 57(1), pp. 208-221.

5. Hasanpour A., Farhadi M., Sedighi K. (2016). Experimental heat transfer and pressure drop study on typical, perforated, V-cut and U-cut twisted tapes in a helically corrugated heat exchanger. International Communications in Heat and Mass Transfer, no. 71, pp. 126-136.

6. Seong-Yeon Yoo, Dong-Seong Park, Min-Ho Chung, Sang-Yun Lee (2002). Heat transfer enhancement for fin-tube heat exchanger using vortex generators. Journal of Mechanical Science and Technology, no. 16, pp. 109-115.

7. Seong Won Hwang, Dong Hwan Kim, June Kee Min, Ji Hwan Jeong (2012). CFD analysis of fin tube heat exchanger with a pair of delta winglet vortex generators. Journal of Mechanical Science and Technology, no. 26, pp. 2949-2958.

8. D. Chalaev, N. Silnyagina, O. Shmatok, A. Nedbailo (2016). Heat transfer enhancement in a corrugated tube heat exchanger. Ukrainian Food Journal, vol. 5, issue 2, pp. 376-386.

9. D. Chalaev, O. Shmatok, T. Grabova, N. Silnyagina. Development of energy-efficient shell and tube heat exchangers for use in geothermal heating systems. $2^{\text {nd }}$ International scientific conference "Chemical technology and engineering". Lviv, June 24-28 ${ }^{\text {th }}$, 2019. Pp. 170-172.

10. Available at: http://www.sanzee.com 